\title{
Research Paper: Effect of Different Intensities of Harsh Reliance of Auditory Stimulation on Static Balance in 5-12 Years Old Children With Cerebral Palsy in Tehran City, Iran
}

\author{
Masomeh Esmailpour Nosar $^{1} \odot$, Seyed Ali Hosseini ${ }^{1,2}{ }^{\circ},{ }^{*}$ Nazila Akbarfahimi $^{1} \odot$, Seyed Farhad Tabatabai Ghomshe ${ }^{3} \odot$, \\ Akbar Biglarian $^{4,5}$ (1)
}

1. Department of Occupational Therapy, University of Social Welfare and Rehabilitation Sciences, Tehran, Iran.

2. Pediatric Neurorehabilitation Research Center, University of Social Welfare and Rehabilitation Sciences, Tehran, Iran.

3. Department of Ergonomics, University of Social Welfare and Rehabilitation Sciences, Tehran, Iran.

4. Department of Biostatistics, University of Social Welfare and Rehabilitation Sciences, Tehran, Iran.

5. Social Determinants of Health Research Center, University of Social Welfare and Rehabilitation Sciences, Tehran, Iran.

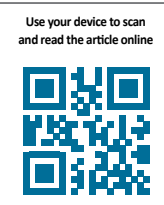

Clttat on Esmailpour Nosar M, Hosseini SA, Akbarfahimi N, Tabatabai Ghomshe SF, Biglarian A. [Effect of Different Intensities of Harsh Reliance of Auditory Stimulation on Static Balance in 5-12 Years Old Children With Cerebral Palsy in Tehran City, Iran (Persian)]. Archives of Rehabilitation. 2018; 19(3):194-205. http://dx.doi.org/10.32598/rj.19.3.194

http://dx.doi.org/10.32598/rj.19.3.194

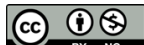

Funding: See Page 202

Received: 10 Apr 2018

Accepted: 28 Aug 2018

Available Online: 01 Oct 2018

\section{A B STRACT}

Objective control dysfunction is a serious problem in children with Cerebral Palsy (CP). This study aimed to investigate the impact of different intensities, harsh reliance of auditory stimulation on static balance in 5-12 years old children with bilateral spastic cerebral palsy.

Materials \& Methods This analytical descriptive study, twenty 5-12 years old children (8 boys and 12 girls, Mean $\pm S D$ age: $7.10 \pm 2.03$ y) with cerebral palsy participated. They were selected by the convenience sampling method. Children with CP were included in the study if they had bilateral spastic CP (diagnosed by neurologist), aged 5 to 12 years, had level I and II CP according to Gross Motor Function Classification System (GMFCS), could stand independently without any support for at least 30 seconds, had no history of surgical treatment or received injection of botulinum toxin within six months prior to the study and not a candidate for such, got scores between 1 to 3 according to Modified Ashworth Scales (MAS) in plantar flexors, and understood verbal comments. They were excluded from the study if they had vision or hearing impairments and uncontrolled epilepsy. Modified Ashworth Scales, Force Plate (during eyes open) and SPARCLE questionnaire were employed to assess muscle tone, static balance, and cognition level, respectively. The central of mass signals were collected at a sampling frequency of $100 \mathrm{~Hz}$, over a period of 30 seconds using a single piezoelectric force platform (Model 9286; Kisler, Switzerland, Bioware 4-0-12). All participants were tested in standing position with eyes opened. The participants stood independently, with bare feet, on the markers in the center of the platform with arms at the sides, while looking straight ahead at the spot in front of them. The participants listened to impure auditory stimulation with harsh and low high intensity on a hard surface and soft force plate for three times. The four tasks were 1. Standing on soft surface while listening to impure auditory stimulation with harsh high intensity; 2 . Standing on soft surface while listening to impure auditory stimulation with harsh low intensity; 3 . Standing on hard surface while listening to impure auditory stimulation with harsh low intensity; and 4 . Standing on hard surface while listening to impure auditory stimulation with harsh high intensity. Between the tasks, a break was provided during which they could rest on the chair for at least 5 minutes. Then the process repeated with auditory stimulation harsh low intensity. The average results across the three times were calculated for each participant. The data were filtered and anterior-posterior, phase plane portrait rate and velocity were measured with MATLAB (R2010a). Data normality distribution was tested using Shapiro-Wilk test which revealed the normality in dis-

\section{* Corresponding Author:}

Nazila Akbarfahimi, PhD

Address: Department of Occupational Therapy, University of Social Welfare and Rehabilitation Sciences, Tehran, Iran. Tel: +98 (21) 22180037

E-Mail: na.akbarfahimi@uswr.ac.ir 
Keywords: Auditory stimulation, Static balance, Spastic cerebral palsy tribution of the data $(P<0.05)$. The independent $t$ test was used to compare the results of anteriorposterior, phase plane portrait rate and velocity for each condition (harsh high intensity on a hard surface, soft force plate, harsh low intensity on a hard and soft surface of force plate). All statistical analyses were performed in SPSS V. 16. The significance level was set at $P<0.05$ for all tests.

Results The effect of harsh high intensity auditory stimulation on the hard and soft surface of the plate anterior -posterior rate $(P<0.001)$ and velocity $(P<0.001)$ were significant. There was no significant effect of harsh low intensity auditory stimulation on the hard and soft surface of the plate anterior-posterior rate $(P=0.38)$ and velocity $(P=0.722)$.

Conclusion According to this study, the harsh high intensity auditory stimulation on hard and soft surfaces with eyes opened affect the speed rate, anterior-posterior, phase plane portrait page, and static balance in children with bilateral spastic CP. Regarding this result, balance programming may improve the static balance in these children. 


\title{
بررسى تأثير تحريك شنيدارى ناملايم با شدت متفاوت بر تعادل ايستاى كودكان فلج مغزى اسياستيك ه تا با ساله در تهران
}

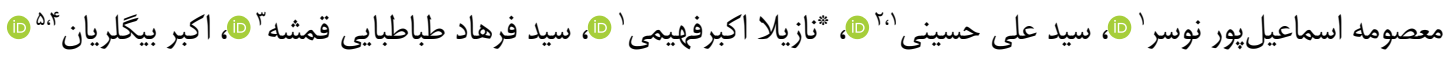 \\ 1 ا- كروه كاردرمانى، دانشگاه علوم بهزيستى و توانبخشى، تهران، ايران.

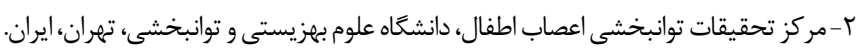

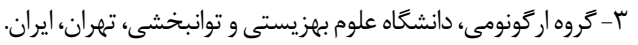

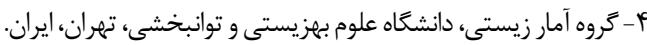

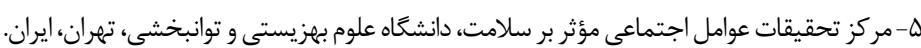

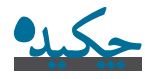

هدف اختلال در كنترل تعادل به عنوان عامل اصلى اختلال در كاركرد كودكان فلج مغزى است. هدف از مطالعه حاضر بررسى تأثير تحريك

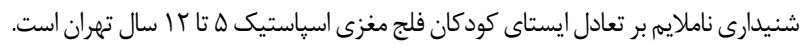

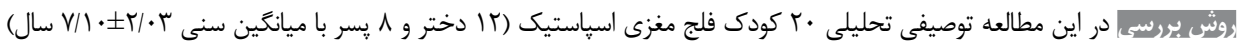

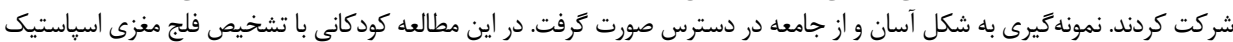

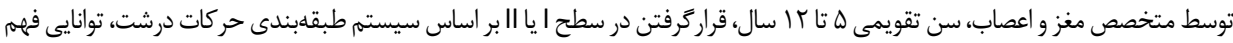

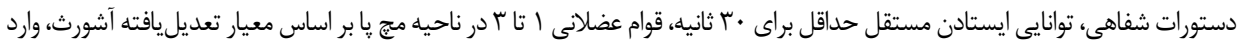

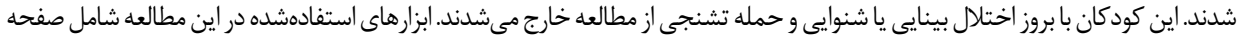

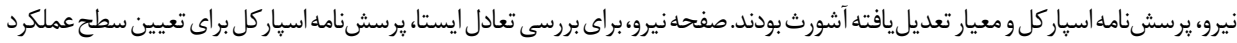

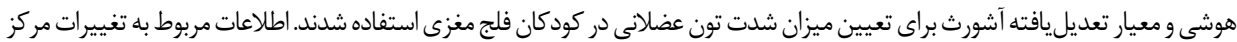

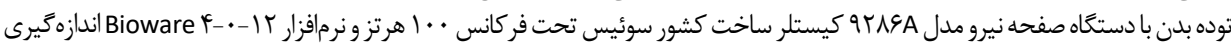

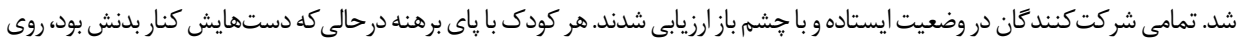

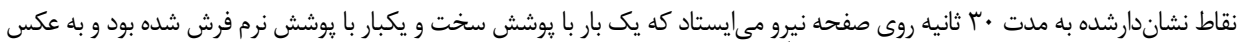

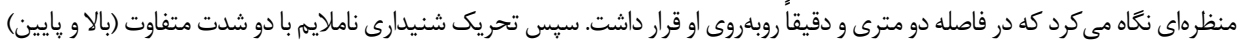

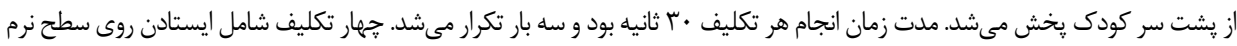

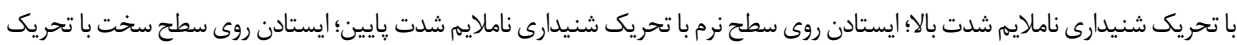

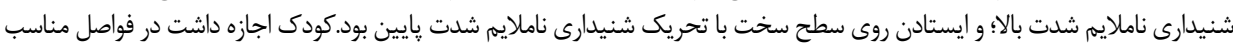

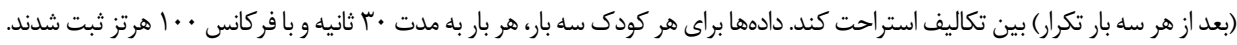

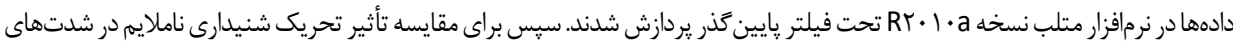

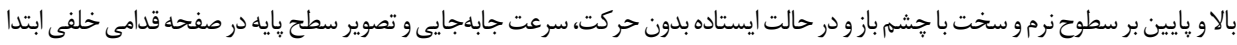

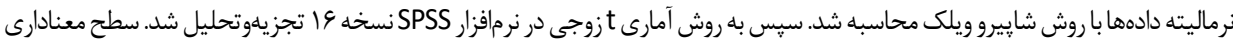

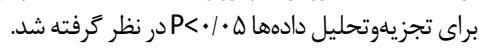

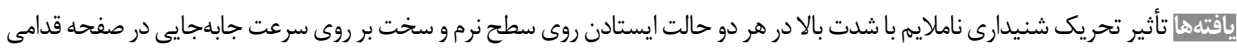

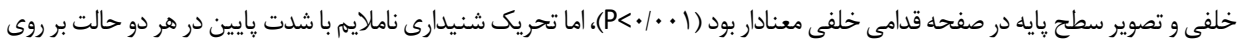

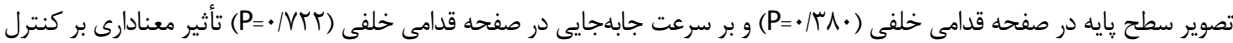
تعادل ايستانداشت.

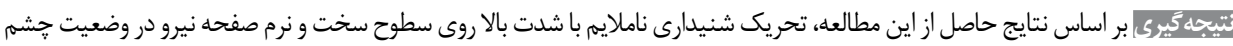

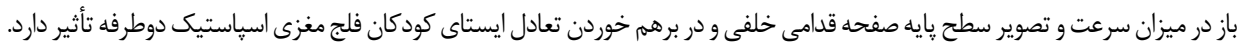

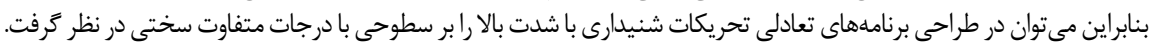

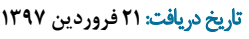

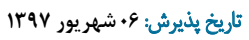

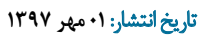

كليدوأزهها:

تحريك شنيدارى، تعادل ايستا، فلج مغزى اسياستيك تعادل

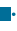

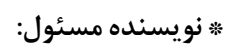

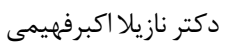

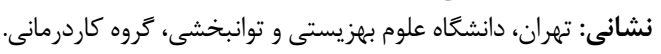

$$
\begin{aligned}
& \text { تلفن: }
\end{aligned}
$$


منبع مهمى براى درك وضعيت و حركت سر در ارتباط با نيروى إنى

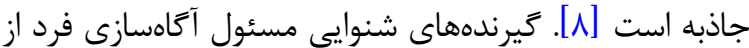

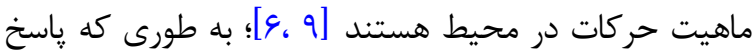

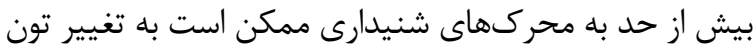

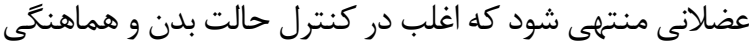

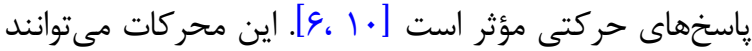

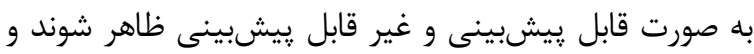

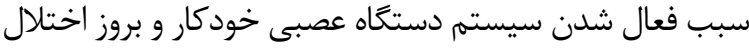

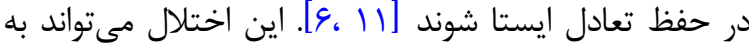

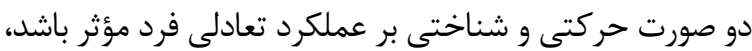

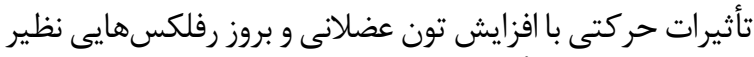

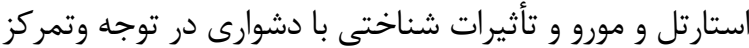

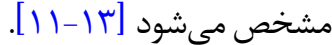

كودكان فلج مغزى اسياستيك تجربه كمى در رد (فيلتريشن)

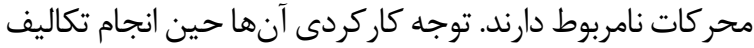

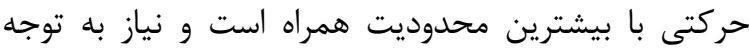

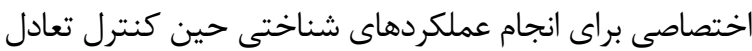

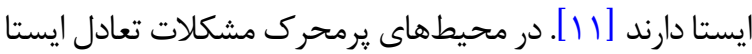

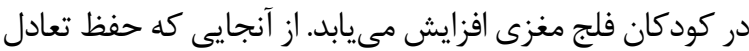

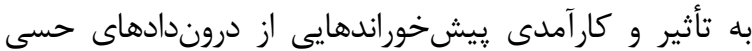

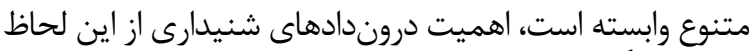
هنوز كاملاً مشخص نشده است [1 11، 11] باوجود آنكه اختلال دركنترل تعادل ايستا يكى از مشخصههاى

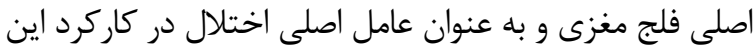

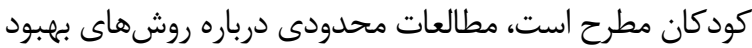

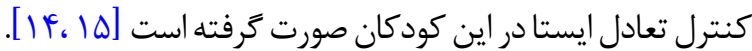

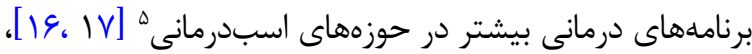

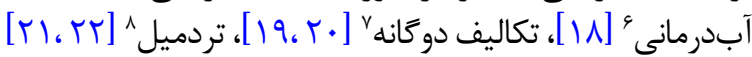

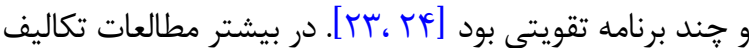

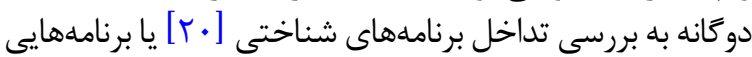

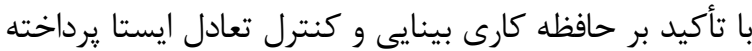

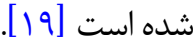

با توجه به علائم كسترده در اين كودكان، ضرورت اجراى

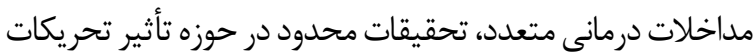

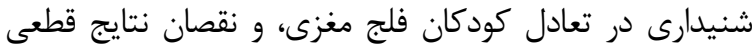

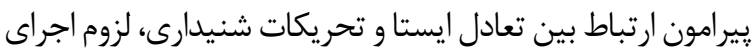

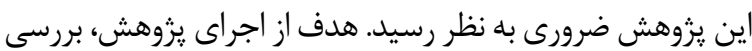

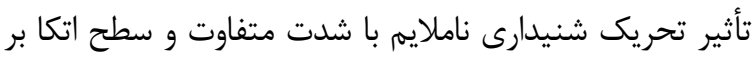

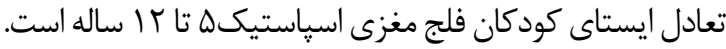

5. Hippotherapy

6. Aquatic therapy

7. Dual task

8. Treadmill therapy otald

فلج مغزى اختلال حركتى و وضعيتى ناشى از صدمه غير من دير

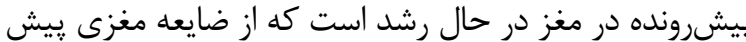

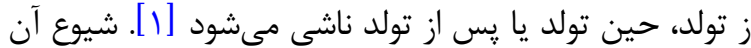

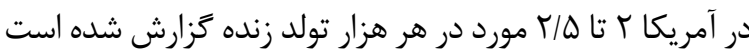

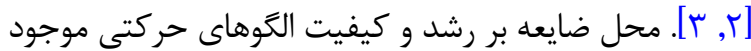

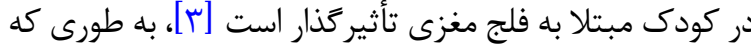

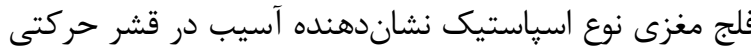

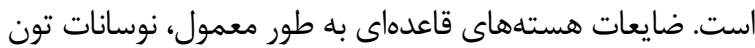

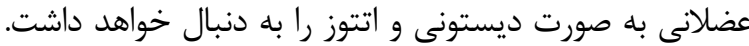

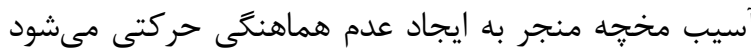

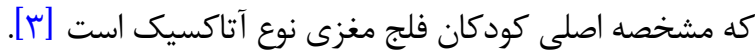

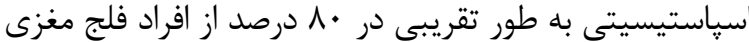

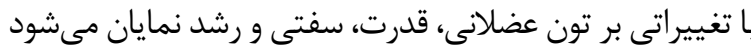

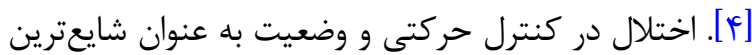

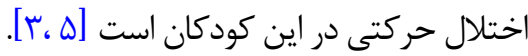

كنترل وضعيت فرايندهاى ييجيده و مداومى است كه به تنظيم

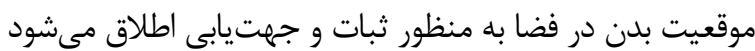

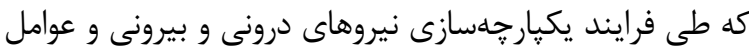

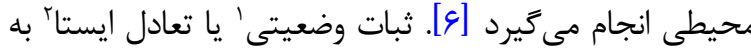

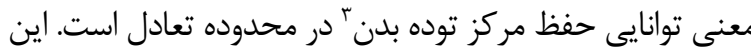

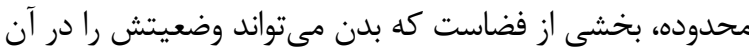

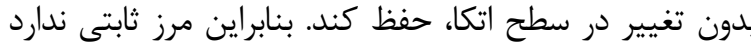

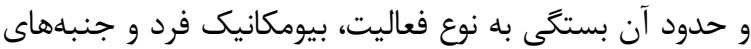

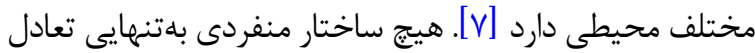
يستا را تحت يوشش قرار نمى دهد، بلكه كنترل تعادل ايستانتيجه

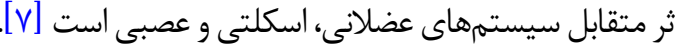
يكى از اجزاى سيستم عصبى در كنترل تعادل ايستا، سيستمهاى

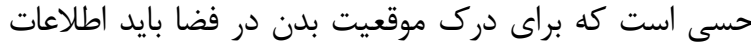

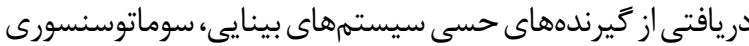

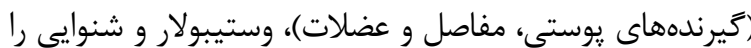

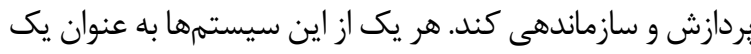

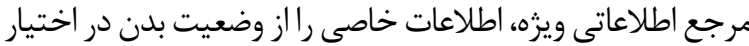

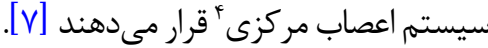

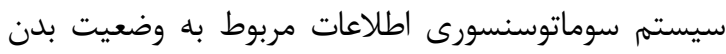

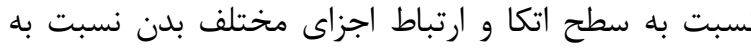

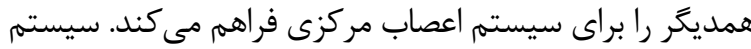

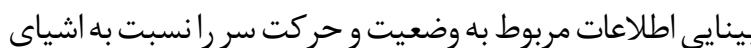
موجود در محيط كزارش مى دهد. اطلاعات سيستم وستيبولار

\footnotetext{
1. Postural stability

2. Static balance

3. Center of Mass (COM)

4. Central Nervous System (CNS)
} 
ارزيابى تخمينى سطح شناختى كودكان طراحى كردند كه

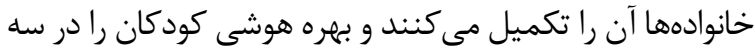

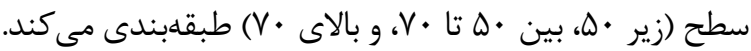

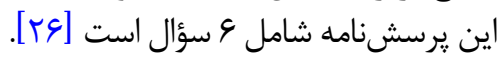

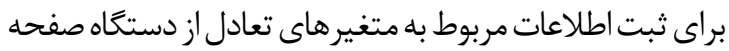

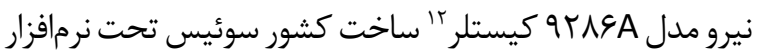

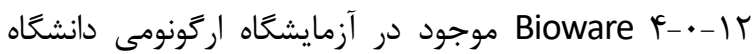

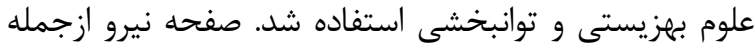
تجهيزات آزمايشكاه بيومكانيك است كه به به اندازمخيرى نيروني

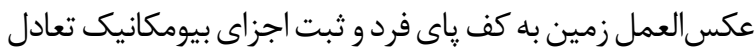

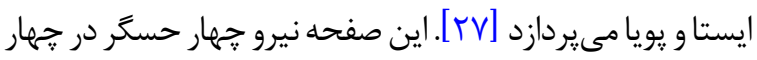

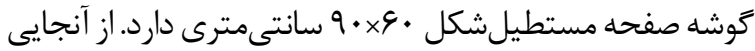

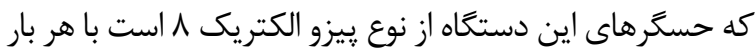

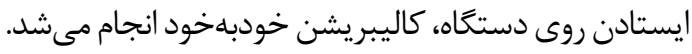
هر كودى با پايى برهنه در حالى كه دستهايش كنار بدنش ندانش

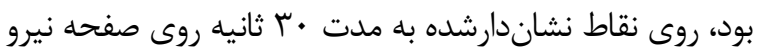

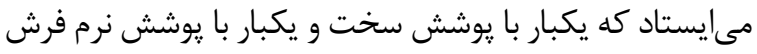

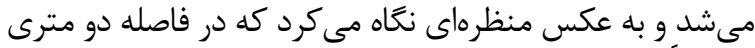

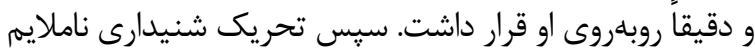

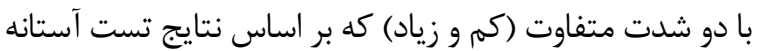

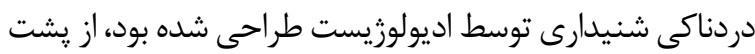

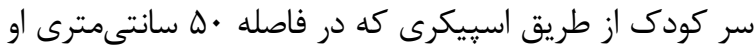

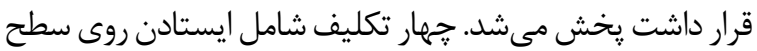

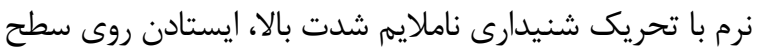

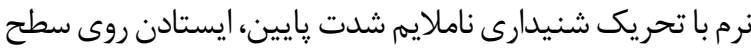

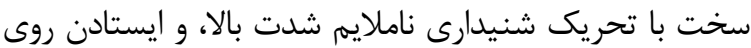

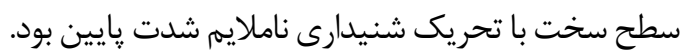
مدت زمان انجام هر تكليف • • ثانيه بود و سه بار تكرار مىشد.

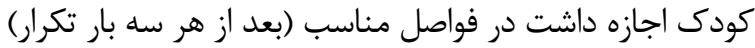

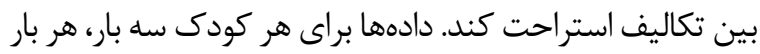

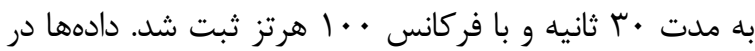

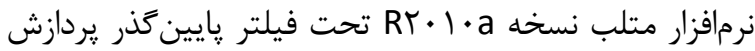

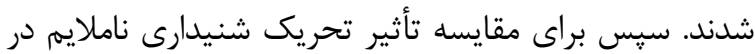

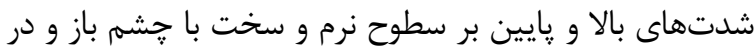

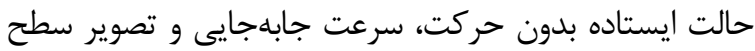

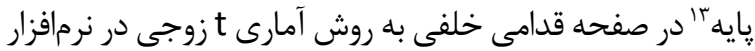

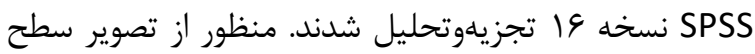

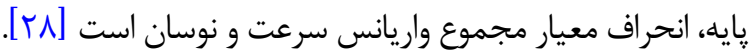

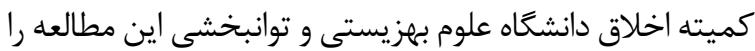

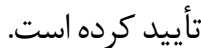

12. Kistler

13. Phase plane
و - مش بروسى

اين تحقيق از نوع توصيفى تحليلى بود كه روى • ب كودى

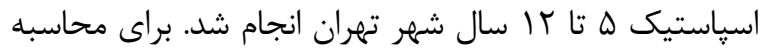

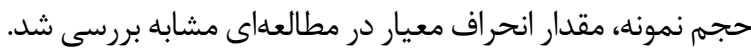

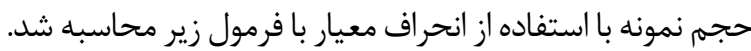

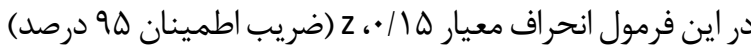

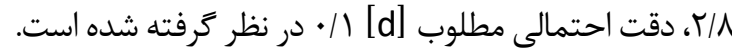

$$
n=\frac{Z^{2} \hat{\sigma}_{p}^{2}}{d^{2}}=\frac{2.8^{2} *(0.15)^{2}}{(0.10)^{2}}=17.64 \square 18 \quad ; \quad Z=Z_{1-\frac{\alpha}{2}}+Z_{1-\beta}
$$

روش نمونهگيرى از نوع در دسترس آسان بود. كودكانى با

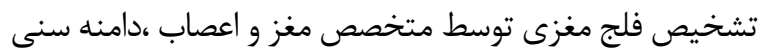

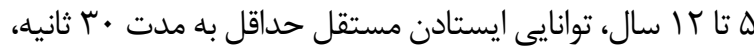

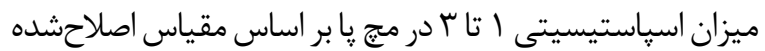

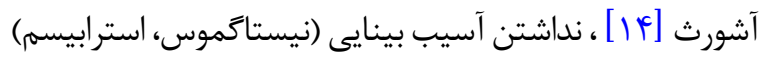

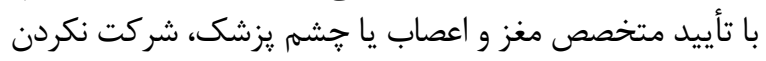

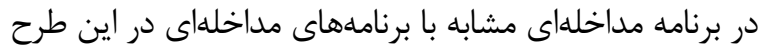

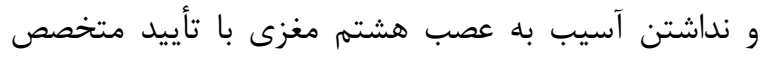

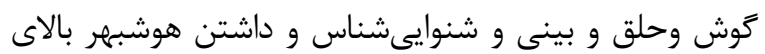

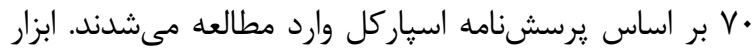

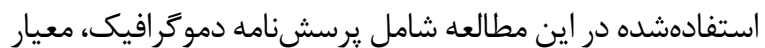

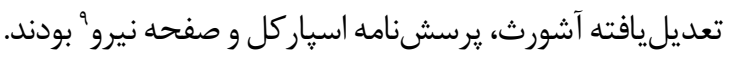
معيار تعديليافته آشورث براى ارزيابى ميزان اسياستيسيتى

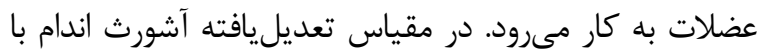

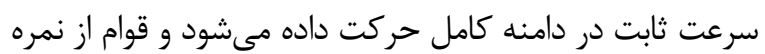

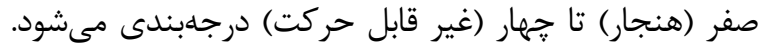

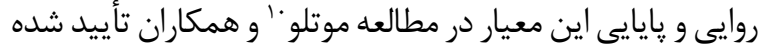

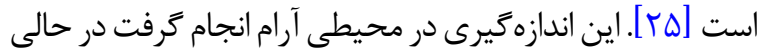

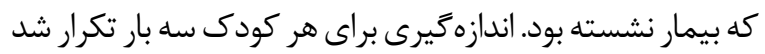

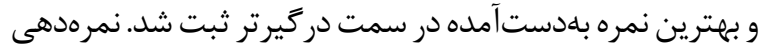

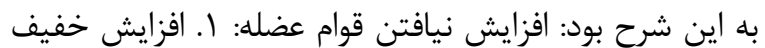

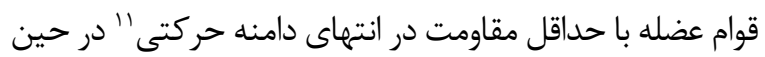

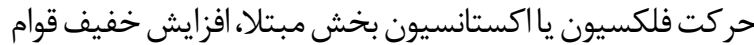

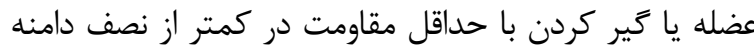

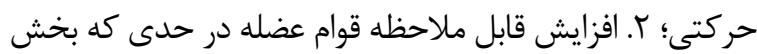

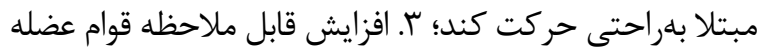

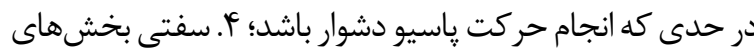
مبتلا در فلكسيون و اكستانسيون. آلن كولور و گروه اسياركل يرسشنامه اسياركل را به منظور

11. Rang of Motion (ROM) 
متغيرها توزيع نرمال داشتند. همانَّونه كه در جدول شماره

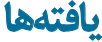

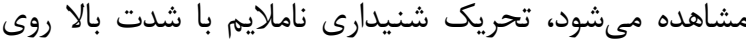

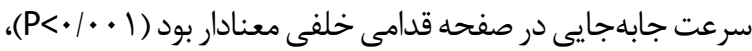

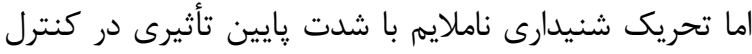

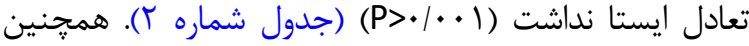

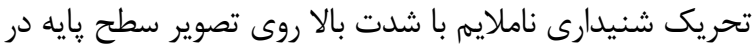

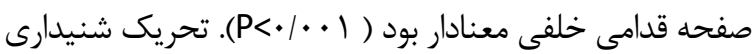

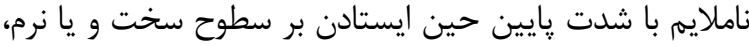

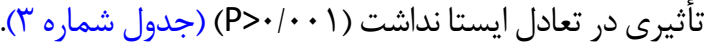

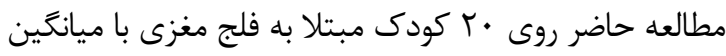

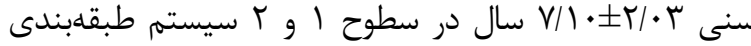

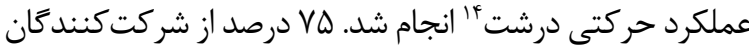

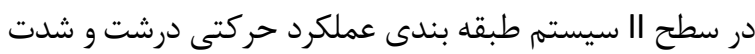

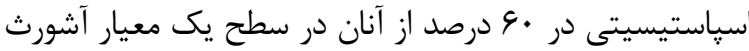

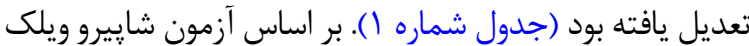

14. Gross Motor Function Classification System (GMFCS)

جدول (. مشخصات جمعيتشناختى شركت كنند

\begin{tabular}{|c|c|c|}
\hline 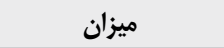 & \multicolumn{2}{|c|}{ متغير } \\
\hline سال & & سن سن \\
\hline 作 & & 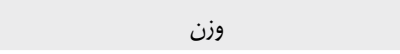 \\
\hline 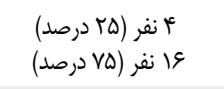 & $\begin{array}{l}\text { سطح س سطح II } \\
\text { II }\end{array}$ & شدت دركيرى (مقياس GMFCS) \\
\hline 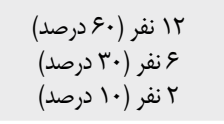 & $\begin{array}{l}1 \\
j \\
1 \\
r\end{array}$ & ميزان سفتى عضلاتى بر اساس مقياس آشورث \\
\hline 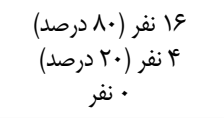 & 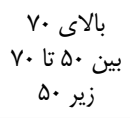 & هوشبهر بر اساس برسشنامه اسياركل \\
\hline 1 ن نفر (•F د درصد) & 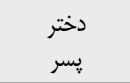 & جنسيت \\
\hline
\end{tabular}

جدول r. ميزان سرعت جابهجايى در وضعيتهاى مختلف شنيدارى و سطوح متفاوت (•rN)

\begin{tabular}{|c|c|c|c|c|c|c|}
\hline$P$ & درجه آزادى & ملاك آزمون & انحراف معيار & ميانكين & & \\
\hline$<\cdot / \cdot \cdot 1$ & $r$. & q/9וץ & $1 . r q$ & $.1 \cdot .9$ & سطح نرم سخح سخت & تحريك شنيدارى ناملايم \\
\hline . IVT & $r$. &.$/ \Delta V T$ & .1 .48 & $\begin{array}{l}\cdot / \cdot 1 \\
. / \cdot v^{c}\end{array}$ & سطح نرم & تحريك شنيدارى ناملايم شدت \\
\hline
\end{tabular}

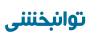

جدول س. ميزان تصوير پايه در وضعيت هاى مختلف شنيدارى و سطوح متفاوت (·r= )

\begin{tabular}{|c|c|c|c|c|c|c|}
\hline$P$ & درجه آزادى & ملاك آزمون & انحراف معيار & ميانكَين & & \\
\hline$<\cdot / . \cdot 1$ & $r$. & $V / V \backslash \Delta$ & /. & $\begin{array}{l}.1 . V \Delta \\
.1 .99\end{array}$ & سطح سخت & تحريك شنيدارى ناهلايم \\
\hline . & $r$. & ./1999 & .1 .19 & $\begin{array}{l}\cdot / \cdot 11 \\
. / \cdot n 1\end{array}$ & سطح نرم سخح & تحريك شنيدارى ناملايم \\
\hline
\end{tabular}

توانبخننى 
عمقى هر دو تروه سنى با افزايش سرعت جابهجايى مركز فنشار

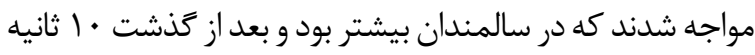

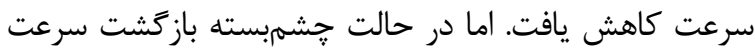

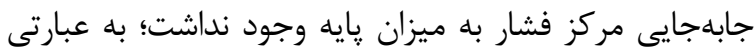

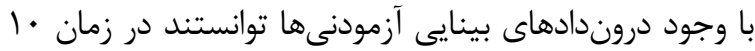

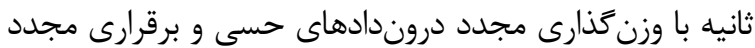

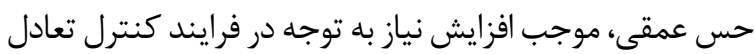

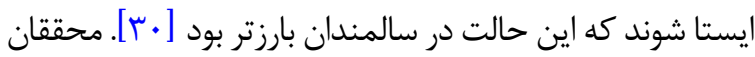

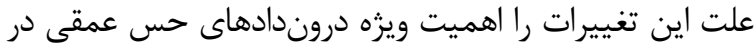

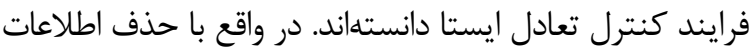

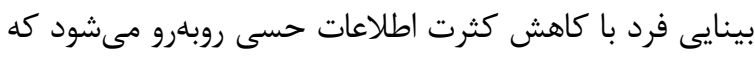

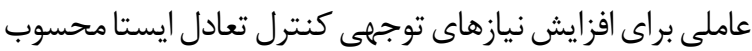

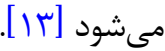

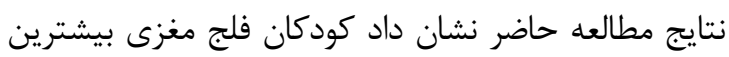

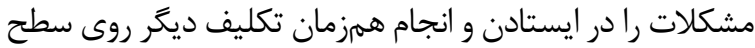

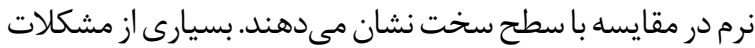

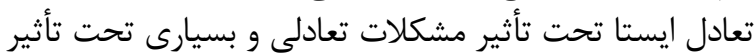

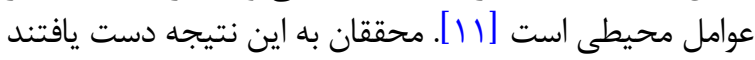

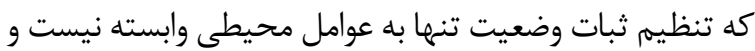

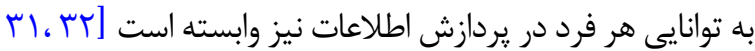

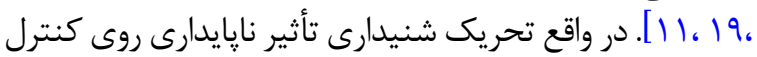

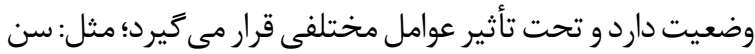

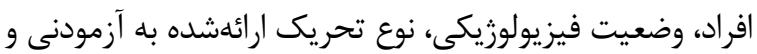

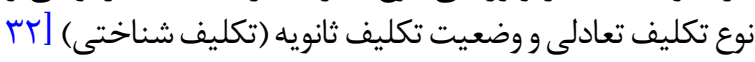

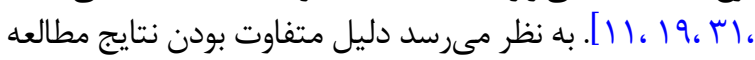

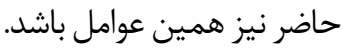

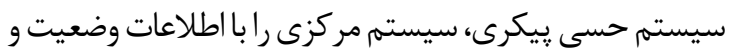

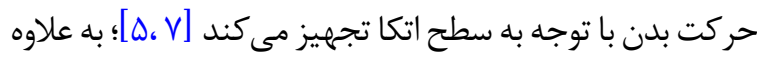

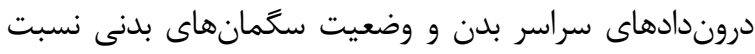

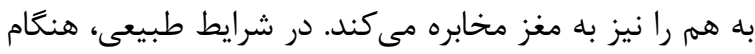

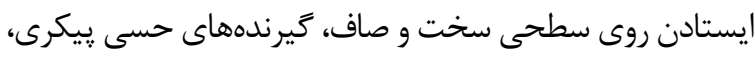

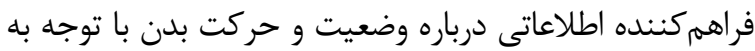

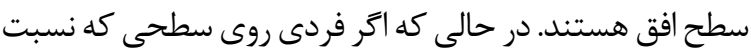

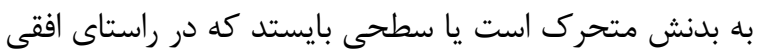

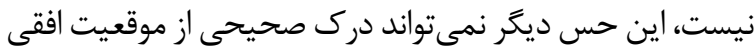

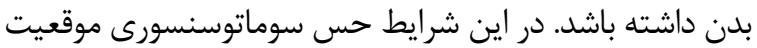

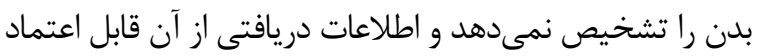

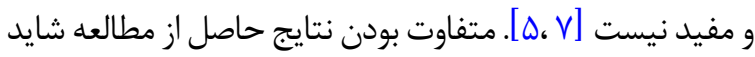

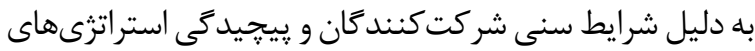

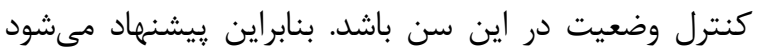

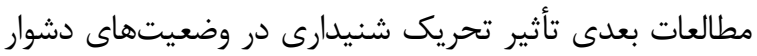

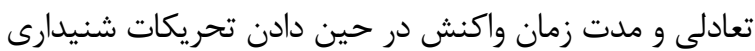

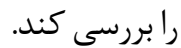

در اين مطالعه نتايج حاصل از تجزيهوتحليل دادهانها نشان داد

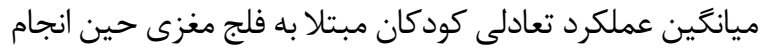

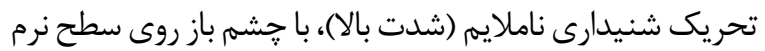

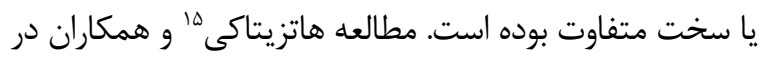

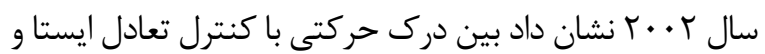

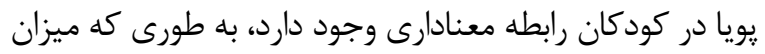

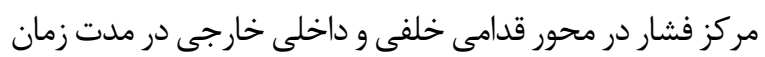

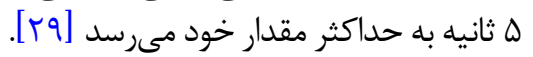

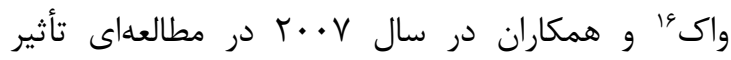

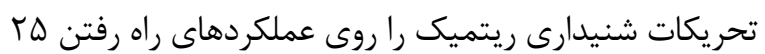

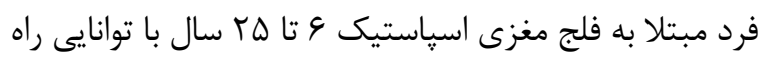

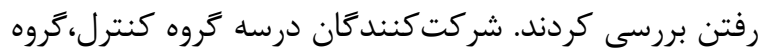

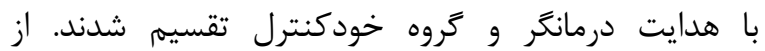

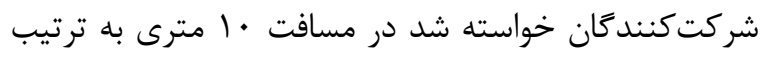

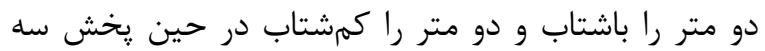
موزيك جاز، مارش و ملايم راه بروند.

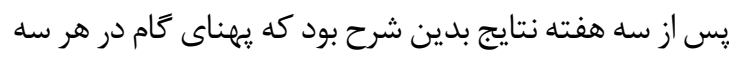

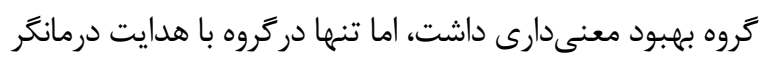

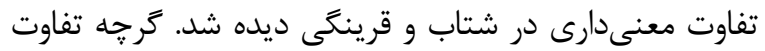

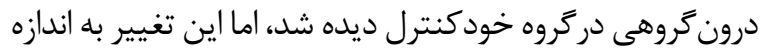

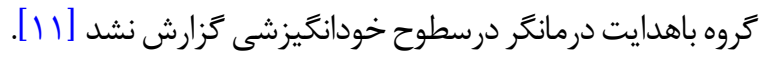

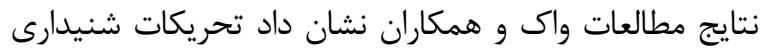

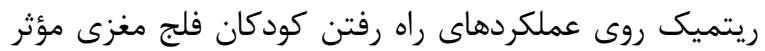

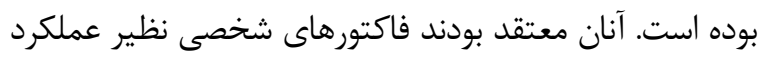

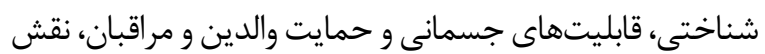

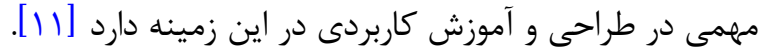

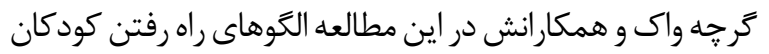

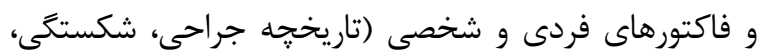

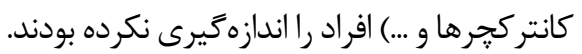

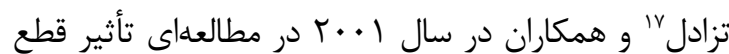

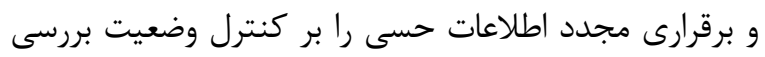

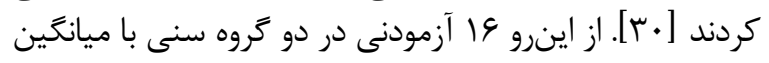

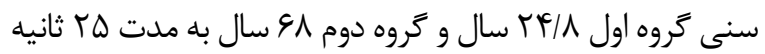

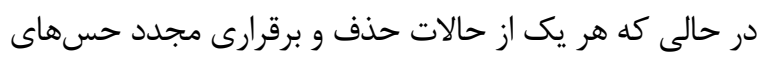

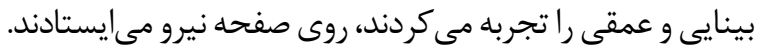

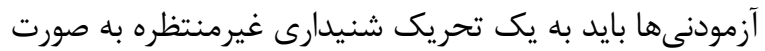
شفاهى ياسخ مى دادند. نتيجه مطالعه نشان داد در زمان برقرارى مجدد اطلاعات حسى ميى 


\section{تثيجه تيرى}

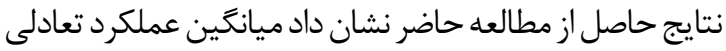

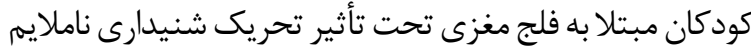

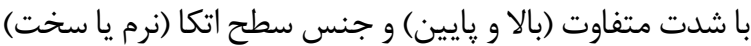

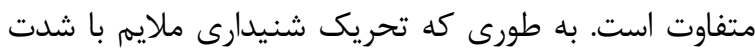

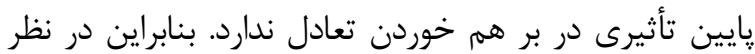

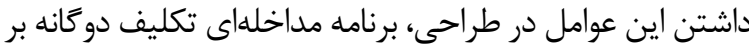

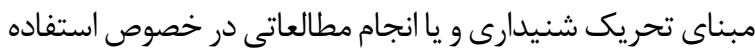

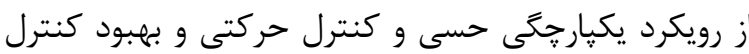

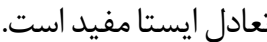

مالاحظات اخلاقي

يميروى از أصول اخلاق يثوهش

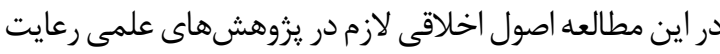

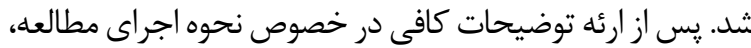

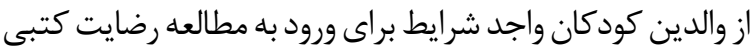

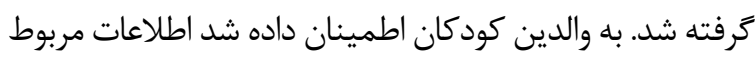

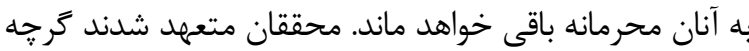

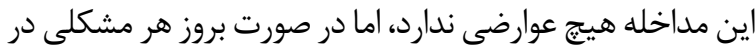

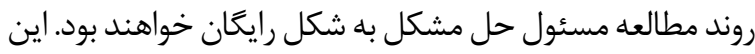
مطالعه هيج هزينه مالى براى شركت كنند مان نداشت.

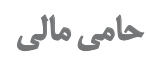

اين مقاله از باياننامه كارشناسى ارشد خانم معصومه

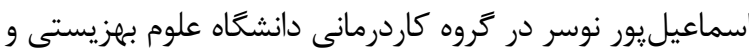

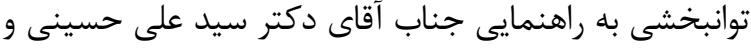

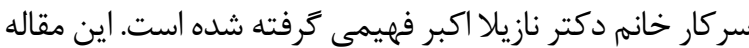

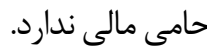

$$
\text { تعارض مناقع }
$$

بنابر اظهار نويسندكان اين مقاله تعارض منافع ندارد.

$$
\text { تشكر و قدردانى }
$$

بدينوسيله از تمام كسانى كه در انجام اين تحقيق ما را يارى

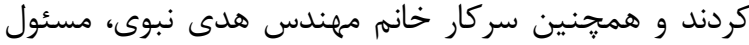

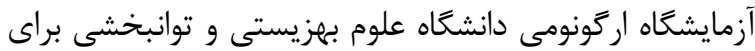

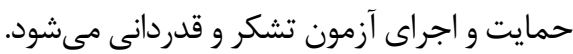




\section{References}

[1] Rosenbaum P, Paneth N, Leviton A, Goldstein M, Bax M, Damiano D, et al. A report: The definition and classification of cerebral palsy April 2006. Developmental Medicine and Child Neurology. 2007; 109:8-14. [PMID]

[2] Odding E, Roebroeck ME, Stam HJ. The epidemiology of cerebral palsy: Incidence, impairments and risk factors. Journal Disability and Rehabilitation. 2006; 28(4):183-91. [DOI:10.1080/09638280500158422] [PMID]

[3] Case-Smith J, O'Brien JC. Occupational therapy for children and adolescents. New York: Elsevier Health Sciences; 2014.

[4] Soleimani F, Vameghi R, Rassafiani M, Akbar Fahimi N, Nobakht Z. Cerebral palsy: Motor types, gross motor function and associated disorders. Iranian Rehabilitation Journal. 2011; 9:21-31.

[5] Akbar Fahimi N, Hosseini SA, Rassafiani M, Farzad M, Haghgoo HA. The reactive postural control in spastic cerebral palsy children. Iranian Rehabilitation Journal. 2012; 10(1):66-74.

[6] Melzer I, Damry E, Landau A, Yagev R. The influence of an auditory-memory attention-demanding task on postural control in blind persons. Clinical Biomechanics. 2011; 26(4):358-62. [DOI:10.1016/j.clinbiomech.2010.11.008] [PMID]

[7] Shumway-Cook A, Woollacott MH. Motor control: Translating research into clinical practice. Philadephia: Lippincott Williams \& Wilkins; 2007.

[8] Maurer C, Mergner T, Bolha B, Hlavacka F. Vestibular, visual, and somatosensory contributions to human control of upright stance. Neuroscience Letters. 2000; 281(2-3):99-102. [DOI:10.1016/ S0304-3940(00)00814-4]

[9] Shumway-Cook A, Woollacott M. Attentional demands and postural control: The effect of sensorycontext. Journals of Gerontology-Biological Sciences and Medical Sciences. 2000; 55(1):M10-16. [DOI:10.1093/gerona/55.1.M10]

[10] Magnusson M, Johansson K, Johansson BB. Sensory stimulation promotes normalization of postural control after stroke. Stroke. 1994; 25(6):1176-80. [DOI:10.1161/01.STR.25.6.1176] [PMID]

[11] Kwak EE. Effect of rhythmic auditory stimulation on gait performance in children with spastic cerebral palsy. Journal of $\mathrm{Mu}-$ sic Therapy. 2007; 44(3):198-216. [DOI:10.1093/jmt/44.3.198] [PMID]

[12] Nobahar Ahari M, Nejati V, Hosseini SA. Attentional Demands of balance under Dual Task conditions in young adults. Iranian Rehabilitation Journal. 2012; 10(16):66-71.

[13] Melzer I, Benjuya N, Kaplanski J. Age-related changes of postural control: Effect of cognitive tasks. Gerontology. 2001; 47(4):189-94. [DOI:10.1159/000052797] [PMID]

[14] Shumway-Cook A, Hutchinson S, Kartin D, Price R, Woollacott M. Effect of balance training on recovery of stability in children with cerebral palsy. Developmental Medicine and Child Neurology. 2003; 45(9):591-602. [DOI:10.1017/S0012162203001099] [PMID]

[15] Novak I, Mcintyre S, Morgan C, Campbell L, Dark L, Morton $\mathrm{N}$, et al. A systematic review of interventions for children with cerebral palsy: State of the evidence. Developmental Medi- cine \& Child Neurology. 2013; 55(10):885-910. [DOI:10.1111/ dmcn.12246] [PMID]

[16] Champagne D, Corriveau H, Dugas C. Effect of hippotherapy on motor proficiency and function in children with cerebral palsy who walk. Physical \& Occupational Therapy in Pediatrics. 2017; 37(1):51-63. [DOI:10.3109/01942638.2015.1129386] [PMID]

[17] Deutz U, Heussen N, Weigt-Usinger K, Leiz S, Raabe C, Polster $T$, et al. Impact of hippotherapy on gross motor function and quality of life in children with bilateral cerebral palsy: A randomized open-label crossover study. Thieme E-Books \& E-Journals-Neuropediatrics. 2018; 49(3):185-92. [DOI:10.1055/s-0038-1635121] [PMID]

[18] Gorter JW, Currie SJ. Aquatic exercise programs for children and adolescents with Cerebral Palsy: What do we know and where do we go. International Journal of Pediatrics. 2011; 2011: 712165 [DOI: 10.1155/2011/712165] [PMID] [PMCID]

[19] Reilly DS, Woollacott MH, van Donkelaar P, Saavedra S. The interaction between executive attention and postural control in dual-task conditions: children with cerebral palsy. Archives of Physical Medicine and Rehabilitation. 2008; 89(5):834-42. [DOI:10.1016/j.apmr.2007.10.023] [PMID]

[20] Samuel A, Solomon MJ, Mohan D. Postural sway in dual-task conditions between spastic diplegic cerebral palsy and typically developing children. International Journal of Health and Rehabilitation Sciences. 2013; 2(2):91-7.

[21] Grecco LA, Tomita SM, Christovão TC, Pasini H, Sampaio LM, Oliveira CS. Effect of treadmill gait training on static and functional balance in children with cerebral palsy: a randomized controlled trial. Brazilian journal of physical therapy. 2013; 17(1):1723. [DOI:10.1590/S1413-35552012005000066]

[22] Sharif-Moradi K, Farah-Pour N. [Comparison of the balance performance of the children with spastic cerebral palsy before and after exercise therapy program (Persian)]. Archives of Rehabilitation. 2006; 7(1):22-8

[23] Ebrahimi Etri A, Asghari L. Comparison of two exercise methods on motor performance and balance in children with spastic cerebral palsy. Journal of Rehabilitation. 2012;13(1):79-87.

[24] Ismailiyan M, Marandi S, Ghardashi Afousi A, Movahedi A, Esfarjany F. Effect of progressive resistance and balance training on upper trunk muscle strength of children with cerebra palsy: A case study. Journal of Rehabilitation. 2016; 17(1):84-93. DOI:10.20286/jrehab-170182]

[25] Mutlu A, Livanelioglu A, Gunel MK. Reliability of Ashworth and modified Ashworth scales in children with spastic cerebral palsy. BMC Musculoskeletal Disorders. 2008; 9(1):44 [DOI:10.1186/1471-2474-9-44] [PMID] [PMCID]

[26] Colver A. Study protocol: SPARCLE: A multi-centre European study of the relationship of environment to participation and quality of life in children with cerebral palsy. BMC Public Health. 2006; 6(1):105. [DOI:10.1186/1471-2458-6-105] [PMID] [PMCID]

[27] Pavão SL, dos Santos AN, Woollacott MH, Rocha NACF. Assessment of postural control in children with cerebral palsy: A review. Research in Developmental Disabilities. 2013; 34(5):136775. [DOI:10.1016/j.ridd.2013.01.034] [PMID] [PMCID] 
[28] Riley PO, Benda BJ, Gill-Body KM, Krebs DE. Phase plane analysis of stability in quiet standing. Journal of Rehabilitation Research and Development. 1995; 32(3):227-35. [PMID]

[29] Hatzitaki V, Zlsi V, Kollias I, Kioumourtzoglou E. Perceptual-motor contributions to static and dynamic balance control in children. Journal of Motor Behavior. 2002; 34(2):161-70. [DOI:10.1080/00222890209601938] [PMID]

[30] Teasdale N, Simoneau M. Attentional demands for postural control: the effects of aging and sensory reintegration. Gait \& Posture. 2001; 14(3):203-10. [DOI:10.1016/S0966-6362(01)00134-5]

[31] Deviterne D, Gauchard GC, Jamet M, Vançon G, Perrin PP. Added cognitive load through rotary auditory stimulation can improve the quality of postural control in the elderly. Brain Research Bulletin. 2005; 64(6):487-92. [DOI:10.1016/j.brainresbull.2004.10.007] [PMID]

[32] Kim SJ, Kwak EE, Park ES, Cho SR. Differential effects of rhythmic auditory stimulation and neurodevelopmental treatment/ bobath on gait patterns in adults with cerebral palsy: A randomized controlled trial. Clinical Rehabilitation. 2012; 26(10):904-14. [DOI:10.1177/0269215511434648] [PMID] 
